\title{
A Short Note on Simple First Order Linear Difference Equations
}

\author{
NiazHussainGhumro \\ AssistantProfessor@Sukkur IBA Sindh Pakistan
}

\begin{abstract}
This paper is entirely devoted to the analysis of linear non-homogeneousdifference equations of dimension one $(n=1)$ and order $p \geq 1$ with constant coefficients:

$$
X_{t}=\phi_{1} X_{t-1}+\phi_{2} X_{t-2}+\ldots . .+\phi_{p} X_{t-p}+U_{t} \text {, where } \phi_{\mathrm{p}} \neq 0 \text {, where } \phi_{1}, \phi_{2}, \ldots, \phi_{p} \text { are given }
$$

constant real numbers. The variableUt representsthe non-autonomous part of the equation and usually denotes some exogenous or independent variable which influences the evolution of Xt over time. Here In this note only discussion would be limited to the linear difference equations $(p=1)$ and their solutions applied in different fields using computer software. There is a linkage of difference equations in the field of Economics and Finance, found in this study.
\end{abstract}

Keywords: Non-homogeneous; linear; difference equation; exogenous; non-autonomous; solution; software; application.

\section{Background:}

The idea of difference equationdates back toprehistoric form in the efforts of the Babylonians as early as 2000 B.C. to extract roots and in more explicit form around 450 B.C. In the Pythagoreans' study the triangular numbers satisfy the difference equation $X_{n}=X_{n-1}+n$, the square numbers the equation $S_{n}=S_{n-1}+n^{2}$ and so forth. They also used a system of difference equations $X_{n}=X_{n-1}+2 Y_{n-1}, \mathrm{Y}_{n}=X_{n-1}+Y_{n-1}$ to produce large solutions of Pell's equation, $X^{2}-2 Y^{2}=1$ and thereby approximations of $\sqrt{2}$. Archimedes (about 250 B.C.) used differenceequationsto compute the perimetersPn and $\mathrm{pn}$ of the circumscribed polygon of $\mathrm{n}$ sides and the inscribed polygon ofn sides,respectively[1].

About 1202, Fibonacci formulated his famous rabbit problem that introduced to the Fibonacci sequence $1,1,2,3,5,8,13,21, \ldots$. However, it appears that the corresponding difference equation $F_{n}=F_{n-2}-F_{n-1}$ was first jotted down by Albert Girard around 1634 and solved by De Moivre in 1730. Bombelliintroduced the equation $\mathrm{Y}_{n}=2+\frac{1}{Y_{n-1}}$ in 1572 , which was matching to the equation $\mathrm{Z}_{n}=1+\frac{1}{Z_{n-1}}$ satisfied by ratios of Fibonacci numbers for approximation to $\sqrt{2}$. Fibonacci also introduced roughly definition for the concept of continued fractions that is intimatelyrelated with difference equations. A more precise definition was formulated byCataldi around 1613. (See Brezinski [2]). The earliest example of a difference equation in twoindices, $\left(\begin{array}{c}n+1 \\ r\end{array}\right)=\left(\begin{array}{l}n \\ r\end{array}\right)+\left(\begin{array}{c}n \\ r-1\end{array}\right)$

or $b_{n+1, r}=b_{n, r}+b_{n, r-1}$ for the binomial coefficients canbe dated back to Chia Hsien (1050) and Omar Khayyam (1100). The methodof recursion became rigorously advanced with the invention of mathematical inductionby Francesco Maurolico in the sixteenth century and with its development byFermat and Pascal in the seventeenth century.

The basic theory of linear difference equations was developed in the eighteenth century by de Moivre, Euler, Lagrange, and others. Generating functions, first used by de Moivre to solve the Fibonacci equation, were exploited by Laplace as part of his work in probability theory.

The idea of using difference equations to approximate solutions of differential equations originated in 1769 with Euler's polygonal method, for which the proof of convergence was given by Cauchy around 1840. The subject seems to have unpleasant until almost the end of the nineteenth century, when Lipschitz, Runge,and Kutta developed improved procedures. The urgent need for numerical approximations during World War I greatly stimulated research in this walk, and the number of publications later exploded with the development of the digital computer. Dahlquist [4] initiated the modern theory of the convergence of multistep methods (see Dahlquist [5] for a historical account). 
The efficient application of linear difference equations to the computation of special functions orginated in 1952 with Miller's algorithm for Bessel functions. Such computations must be done with care because of the possibility of explosive roundoff error, as illustrated by the cautionary example of Gautschi [6]. Wimp[10] discusses the development of this method and related algorithms due to Olver, Clenshaw, Gautschi, Wimp, and others. Further development of the theory of linear difference equations has brought the subject to a state comparable to that of linear differential equations (see Hartman [9], and Ahlbrandt and Peterson [1]).

During the 1950's, several ecologists used simple nonlinear difference equations, including the logistic equation, to study the change in populations from one year (or season) to the next with the emphasis on the stability of the iteration. However, in the early 1970's May investigated the variety of complex behavior exhibited by the logistic equation and pondered the possible relationship of this behavior to observed fluctuations in real populations. Additional discoveries about the logistic and related equations were soon made by York, Sarkovskii, Feigenbaum, and others (see Gleick [7]), and the remarkably intricate properties of these equations led totheir becoming a focus in the developing area of chaotic dynamical systems. The excitement of these discoveries attracted the attention of researchers who attemptedto apply the results to fields such as economics,finance to medicine etc.

\section{Introduction:}

In this paper the model is focused whose endogenous variables are constant as well as changing over time which covers theory and applications in the area of difference equations. Difference equations are the first step in the journey towards being able to solve dynamic optimization problems. A difference equation links the value of an endogenous variable in a given time period to its value in other time periods as well as to other exogenous variables. The solution to many dynamic optimization problems relates the value of variables to their past values. So the basic description of the behavior of a variable is different; it is no longer merely a function of other contemporaneous exogenous variables but is also a function of time-lagged values of itself, and in some cases a function of time itself. Many economic variables,known as 'stock' variables behave in this fashion. For example, the capital stock in a country or a firm is dependent not just on current variables like interest rates, investment, openness, corruption etc. but also is a function of how much capital remained in the last period.

This study is different from differential equations; which are distinguished from difference equations by the fact that the changes in variables happen continuously instead of discretely as in the difference equations case. This study is divided further following sections as Solving first order linear difference equations, Dynamics of first order linear difference equations, Phase diagrams, Software touch and conclusion.

\section{Solving First Order Linear Difference Equations:}

A first order linear difference equation is one that relates the value of a variable at aparticular time in a linear fashion to its value in the previous period as well as to otherexogenous variables. In other words a first order linear difference equation is of the form $X_{t}=\phi X_{t-1}+f(t)$, where $\mathrm{f}(\mathrm{t})$ is an exogenous variables. A solution to a first order difference equation is a sequence of values $\left\{X_{t}\right\}, \quad$ expressed as afunction of time and of the exogenous variables. In other words one needs to find a time path for the variable $\mathrm{X}$ that is consistent with the difference equation.

An exact solution to a difference equation also requires that has a single value of the endogenous variable: whether it be an initial condition (a value at the beginning) or a terminal condition (a value at the end). An example of a first order difference equation will be of the form $X_{t}=\phi X_{t-1}$ with initial condition $X_{0}=10$.

\section{Method 1: The General Method}

The general method, as the name suggests, provides a systematic general framework forsolving firstorder difference equations. Suppose it is given a general first order differenceequation of the form

$$
X_{t}=\phi X_{t-1}+\beta
$$

the solution to this difference equation consists of two parts: a part named the particular solution, denoted $X_{P}$ which is any solution to the first order difference equation given (1) and a part named the homogenous solution, denoted $X_{H}$ which solves the differenceequation

$$
X_{t}=\phi X_{t-1}
$$


Consider by thinking about the homogenous solution, i.e. the solution to (2) Since at each stage $\mathrm{X}$ is $\phi$ times the previous value, the solution to this would be of the form

$$
X_{H}=\phi^{t} A
$$

where $\mathrm{A}$ is a constant. The ' $\mathrm{A}$ ' term is important because it considers all possible solutions to (2).

Now ponder about the particular solution, which can be any solution to the equation(1). Since any solution is allowed, it can be, for example, traced out a solution that sets all the

$\mathrm{X}$ 's equal. If onesets $X=\phi X+\beta$, gets the solution

$$
X_{P}=\frac{\beta}{1-\phi}
$$

Combining (3) and (4), the general solution would be

$$
X_{t}=X_{P}+X_{H}
$$

Plugging the values in (5) one gets

$$
X_{t}=\phi^{t} A+\frac{\beta}{1-\phi} \text {. }
$$

Since it is known the value of $X_{0}$,can be pinned down the value of A. Setting $\mathrm{t}=0$ one gets $X_{0}=A+\frac{\beta}{1-\phi} \Rightarrow A=X_{0}-\frac{\beta}{1-\phi}$. So the solution to the difference equation is of the form:

$X_{t}=\phi^{t}\left[X_{0}-\frac{\beta}{1-\phi}\right]+\frac{\beta}{1-\phi} \equiv \phi^{t} X_{0}+\beta\left[\frac{1-\phi^{t}}{1-\phi}\right]$

The general method only works for $|\phi| \neq 1$. If $\phi=1$ or -1 .There are different techniques to solve difference equation[11].

Example: It can be illustrated the general method with a specific numerical example: Solve the

Difference equation $X_{t}=0.2 X_{t-1}+4$ where $X_{0}=10$ using the general method. Let's begin by thinkingabout the homogenous solution $X_{t}=0.2 X_{t-1}$. The solution is of the form $X_{t}=0.2^{t} \mathrm{~A}$ where

A is a constant.The particular solution is the solution to $\mathrm{X}=0.2 \mathrm{X}+4 \Rightarrow \mathrm{X}=5$, So $X_{P}=5$. Combining we get $X_{t}=0.2^{t} A+5$. Since one knows the value of $X_{0}$, can pin down the value of Aby setting $\mathrm{t}=0$ to get $X_{0}=$ $\mathrm{A}+5=10 \Rightarrow \mathrm{A}=5$. So the solution to the difference equation is of the form:

$$
X_{t}=0.2^{t}(5)+5
$$

\section{Method 2: Solving by Repeated Iteration}

A difference equation can be solved fairly easily through repeated iteration. Consider thesimple example $X_{t}=\phi X_{t-1}+\beta$ with $X_{0}$ given. one can rewrite this as

$$
\begin{aligned}
X_{t} & =\phi X_{t-1}+\beta \\
X_{t} & =\phi\left(\phi X_{t-2}+\beta\right)+\beta=\phi^{2} X_{t-2}+\phi \beta+\beta \\
& =\phi\left(\phi X_{t-3}+\beta\right)+\phi \beta+\beta \\
\cdot & \\
& =\phi^{t} X_{0}+\beta\left(1+\phi+\phi^{2}+\ldots+\phi^{t-1}\right) \\
& =\phi^{t} X_{0}+\beta\left(\frac{1-\phi^{t}}{1-\phi}\right) \ldots \ldots \ldots \ldots . . . .(8)
\end{aligned}
$$

It can be also handled the special cases $\phi=1$ and $\phi=-1$ using this technique. 
In the case of $\phi=1$, the difference equation $X_{t}=X_{t-1}+\beta$ with $X_{0}$ given can be solved as

$$
\begin{aligned}
X_{t} & =X_{t-1}+\beta \\
X_{t} & =\left(X_{t-2}+\beta\right)+\beta=X_{t-2}+\beta+\beta=X_{t-2}+2 \beta \\
& =\left(X_{t-3}+\beta\right)+2 \beta=X_{t-3}+\beta+2 \beta=X_{t-3}+3 \beta
\end{aligned}
$$

In the case of $\phi=-1$, the difference equation $X_{t}=-X_{t-1}+\beta$ with $X_{0}$ given can be solved as

$$
\begin{aligned}
& \begin{aligned}
X_{t} & =-X_{t-1}+\beta \\
X_{t} & =-\left(-X_{t-2}+\beta\right)+\beta=X_{t-2}-\beta+\beta=X_{t-2} \\
& =\left(-X_{t-3}+\beta\right)=-X_{t-3}+\beta=-X_{t-3}+\beta \\
& =-\left(-X_{t-4}+\beta\right)+\beta=X_{t-4}-\beta+\beta=X_{t-4}
\end{aligned} \\
& \cdot \\
& X_{t}=\left\{\begin{array}{cc}
X_{0} & \text { if } t \text { is even } \\
\beta-X_{0} & \text { if } t \text { is odd }
\end{array}\right.
\end{aligned}
$$

\section{Dynamics Of First Order Linear Difference Equations}

Having solved for the time path, it can be examined the dynamic behavior of a variable (i.e. itsbehavior over time).This study tries to examine the stability of the difference equation as well as itssteady state value. The steady state value is a value at which the endogenous variable exhibits no dynamic adjustment. In other words, given the difference equation $X_{t}=\phi X_{t-1}+\beta$, a steady state

value $\stackrel{*}{X}$ exhibits the property that $\stackrel{*}{X}_{t}=\stackrel{*}{*}_{t-1}$. A difference equation can have zero, one ormore (in the case of non-linear difference equations) steady state values.

In the above difference equation, the steady state value is therefore ${ }^{X}=\stackrel{*}{X}+\beta \Rightarrow \stackrel{*}{X}=\frac{\beta}{1-\phi}$.

One can verify this by showing that if $X_{t}=\frac{\beta}{1-\phi} \Rightarrow X_{t+1}+\frac{\beta}{1-\phi}$.

A difference equation is said to be stable if $t \rightarrow \infty X_{t}$ is a finite value. If $t \rightarrow \infty \lim _{t}$ does not exist then the difference equation is said to be unstable. A stable difference equation will converge to a steady state value. For example, the differenceequation $X_{t}=0.2 X_{t-1}+4$ is stable because we can show using the solution $X_{t}=(0.2)^{t}(5)+5$ that $t \rightarrow \infty X_{t}=5$. For the general form $X_{t}=\phi X_{t-1}+\beta$, where $\phi \neq 1$, which has the 
solution $X_{t}=\phi^{t} X_{0}+\beta\left[\frac{1-\phi^{t}}{1-\phi}\right]$,it will only be stable if $|\phi|<1$ in which case the steady state will be $t \rightarrow \infty X_{t}=t \stackrel{\operatorname{Lim}}{\rightarrow} \infty\left[\phi^{t} X_{0}+\beta\left[\frac{1-\phi^{t}}{1-\phi}\right]\right]=\frac{\beta}{1-\phi}$.

This situation is viewed in the figure-1.[12]

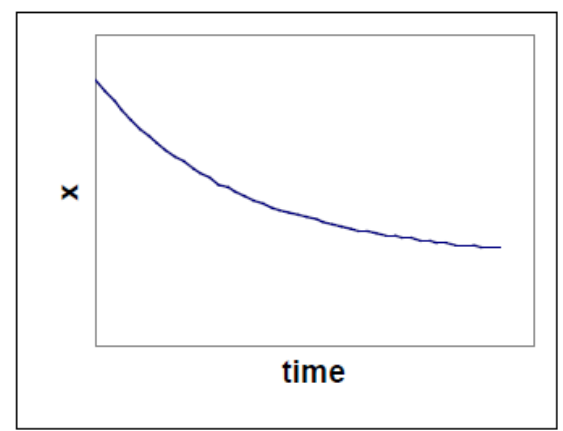

Figure 1: $0<\phi<1$ : Stable, not oscillating

The equation will be unstable if $\phi<-1$ or $\phi>1$ Why? Well if $\phi<-1$ or $\phi>1$ then $t \rightarrow \infty X_{t}=t \rightarrow \infty\left[\phi^{t} X_{0}+\beta\left[\frac{1-\phi^{t}}{1-\phi}\right]\right]$ is undefined, approaching either $\infty$ or $-\infty$.

This situation is viewed in the figure- 2 and 3 [12].

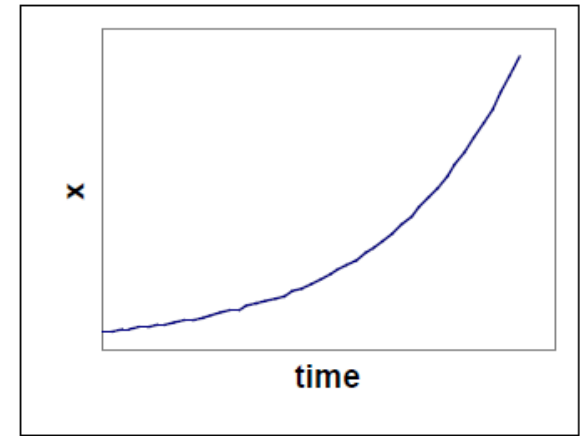

Figure 2: : $\phi>1$ : Unstable, not oscillating

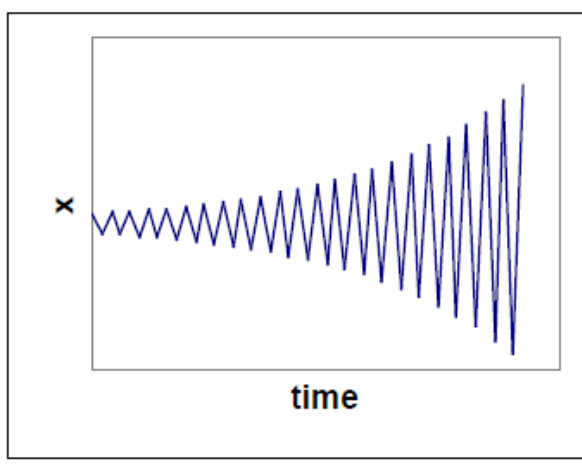

Figure 3: $\phi<-1$ : Unstable, Oscillating

If $\phi=1$, the solution is $X_{t}=X_{0}+\beta t$. This diverges unless $\beta=0$. So except in the extremelyuninteresting case where all the Xs are the same (hence not even a difference equation perse), the difference equation with $\phi=1$ diverges.

If $\phi=-1$, the solution is $\mathrm{X}_{\mathrm{t}}=\left\{\begin{array}{cc}X_{0} & \text { if } t \text { is even } \\ -X_{0}+\beta & \text { if } t \text { is odd }\end{array}\right.$

one can see that this sequence will oscillate between two values as $\mathrm{t}$ gets larger. So thesequence will not converge unless $X_{0}=\frac{\beta}{2}$ Note, also, that this difference equation has asteady state at $\stackrel{*}{X}=\frac{\beta}{2}$ even though it is not stable.

The equation is stable and oscillating if $-1<\phi<0$ and can be viewed in figure-4 [12] 


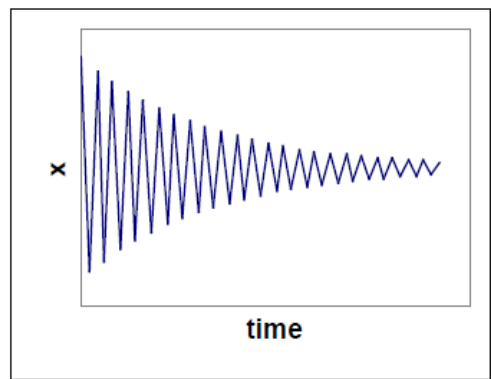

Figure 4: $1<\phi<0$ : Stable, Oscillating

\section{Phase Diagrams}

Sometimes, one analyzes the dynamics of the first order difference equation without solvingit explicitly using either the iterative method or the general method. It can be done this using adiagram known as the 'Phase Diagram'. The phase diagram is a simple graph with Xt and Xt-1 on the two axes. The graph shows two curves. The first plots the difference equation, the second is a 45 degree line representing the steady state (recall that the steady state is where $\mathrm{Xt}=\mathrm{Xt}-1)$.

Consider the simple difference equation solved for earlier: $\mathrm{Xt}=0.2 \mathrm{Xt}-1+4$ where $\mathrm{X} 0=10$.

The phase diagram would look as shown in figure-5.

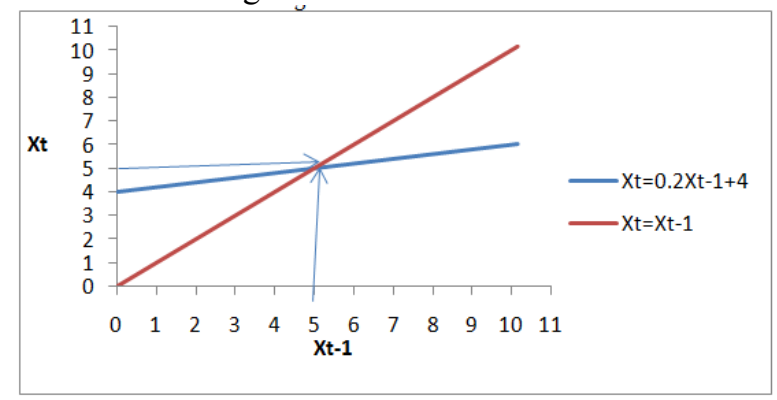

Figure 5: showing the existence of equilibrium

The curves intersect at $\mathrm{Xt}, \mathrm{Xt}-1)=(5,5)$, the steady state of the model. Think of the horizontalaxis as representing yesterday's value and the vertical axis today's value of y. We can then use the phase diagram to trace out the stability or instability of the differenceequation. If yesterday's value was the starting value of X0 = 10 , then the difference equationtells us that today's value will be 6 . So we move to the point $(10,6)$ on the line representingthe difference equation. This period's $\mathrm{Xt}=6$ becomes next period's $\mathrm{Xt}-1$ so we then move tothe mirror point $(6,6)$ on the 45 degree line. We repeat this process until we converge to avalue of $(5,5)$.Perhaps another example will help clarify the situation. Suppose the difference equation hadinstead been $\mathrm{Xt}=0.2 \mathrm{Xt}$ $1+4$, with a starting value of $\mathrm{X} 0=10$. In this case the intersectionwill be at 10/3. The phase diagram would look like as shown in figure-6.

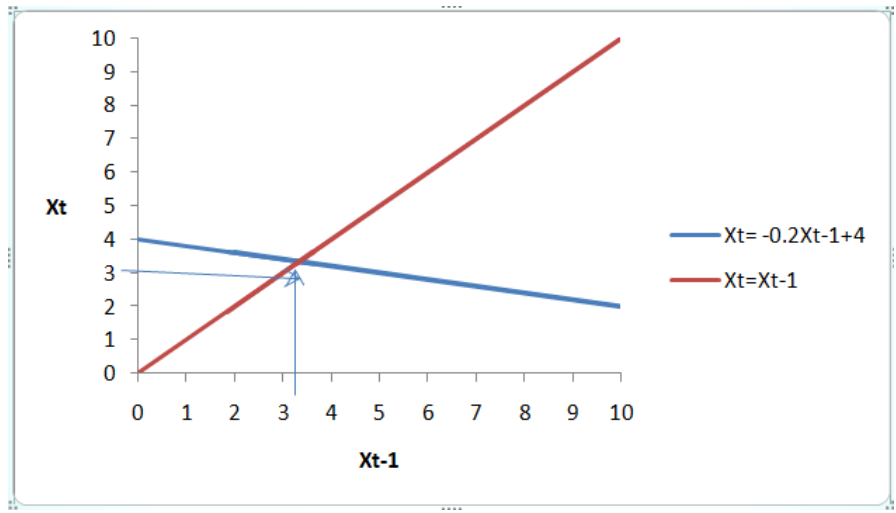

Figure 6: showing the existence of equilibrium

The phase diagram traces out the stability of difference equations.If yesterday's valuewas the starting value of $X_{0}=10$, then today's value will be 2 . So one should move to the point $(10,2)$ 
on the line representing the difference equation. This period's $\mathrm{Xt}=2$ becomes next period's

Xt- 1 so one then moves to the mirror point $(2,2)$ on the 45 degree line. One should repeat this processuntil it converges, reaching a value of $(10 / 3,10 / 3)$.

Finally, consider the simple difference equation $X_{t}=2 X_{t-1}+4$ where $\mathrm{X} 0=0$. The phasediagram would look as shown in figure-7, with the curves intersecting at $(-4,-4)$.

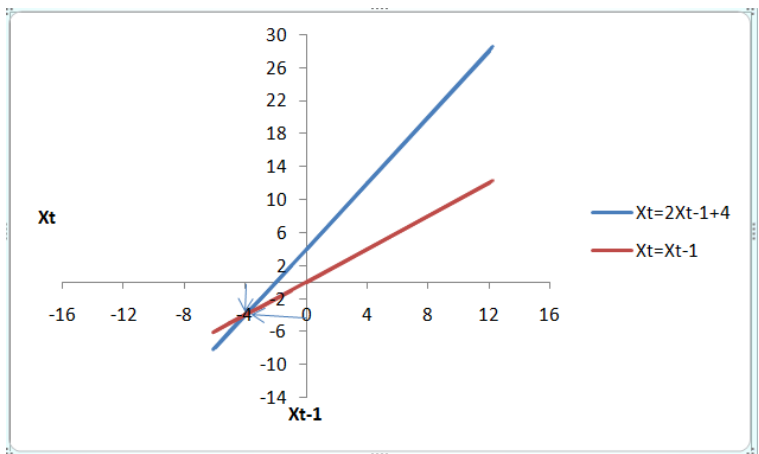

Figure 7: showing that equilibrium does not exist

The phase diagram traces out the instability of the difference equation. Given a starting valueof 0 , the value next period becomes 4 , so one moves to the point $(0,4)$ on the line representingthe difference equation. This period's $\mathrm{Xt}=2$ becomes next period's Xt- 1 so one then moves tothe mirror point $(4,4)$ on the 45 degree line. The following period, $\mathrm{y}$ increases to 12 , takingus to $(4,12)$. Note that we no longer converge; the values of $\mathrm{X}$ become progressively largerand larger. What about the intersection point $X=-4$. That represents an unstable equilibrium, in thatif the initial condition was $\mathrm{X} 0=-4$ one would stay there but for any other starting value of $\mathrm{y}$, graph would quickly be on a divergent trajectory that took us to either $\infty$ or $-\infty$.

\section{Method 3: The MATLAB Method}

It is looked at above how to solve first order difference equations: either by using theiterative method or by the general method and also examined the dynamics of first orderdifference equations, focusing on the stability of the difference equation in the long run through phase diagrams. This study focuses on the applications of difference equations in Economics and Finance through MATLAB.

Let us create an example in investment analysis let Initial value is Rs. 10000 for rate $9.5 \%$ annually after 30 years the account value accumulation would be in the form of Difference equation as

$$
A(t)=\left(1+\frac{r}{100}\right) A(t-1), \mathrm{A}(0)=10,000 \text { has solution } A(t)=\left(1+\frac{r}{100}\right)^{t} A(0) \text { and in MATLAB. }
$$

$\mathbf{t}=[1: 31]$

$\mathrm{A}(1)=10000$;

for $m=2: 31$;

$A(m)=1.095 * A(m-1)$;

end

A(31)

The output would be as underRs.152203.1271.

If contribution ' $c$ ' is added Rs. 1000 at the end of each year in the account with the same rate, possesses the $A(t)=\left(1+\frac{r}{100}\right) A(t-1)+C, \mathrm{~A}(0)=10,000$ and $\mathrm{C}=1000$ and the MATLAB can perform this task as under in form of annuity

$\mathbf{t}=[\mathbf{1 : 3 1}]$;

$A(1)=10000$;

$c=1000$;

for $m=2: 31$;

$A(m)=(1+.095) * A(m-1)+c ;$

end

$\mathbf{A}(31)$

The output would be as under Rs.301890.6293. 
Problems of Loan amortization can be translated in the form of difference equations. One will run into this phenomena again and again in life as one buys a house, a car at each stage wondering how in the world the payment number is derived, and what factors influence it most.

Suppose an individual has borrowed $\mathrm{P}$ dollars at an interest rate of $\mathrm{r}$ per period to be repaid back over $\mathbf{N}$ periods and let $\mathrm{B}_{t}$ be the balance on the loan at time $\mathbf{t}$ and $\mathbf{x b e}$ the size of the payment, which is due

at the end of the period. One can quickly see that the evolution of the outstandingloan balance is given by $B(t)=(1+r) B(t-1)-x$ with two additional conditions - an initial one and a terminal one - which one knows hold, $\mathrm{B}(0)=\mathrm{P}$ and $\mathrm{B}(\mathrm{N})=0$.

So if one borrowed Rs. 20,000 to be repaid over 40 quarters at an interest rate of $1.75 \%$ per quarter, with quarterly installment of Rs. 699.44, then remaining balance at specific quarter will be $B(t)=(1+r) B(t-1)-x$ where $\mathrm{B}(0)=\mathrm{P}=$ Rs. 20,000 and $\mathrm{x}=699.44$. This can be transformed into MATLAB as under.

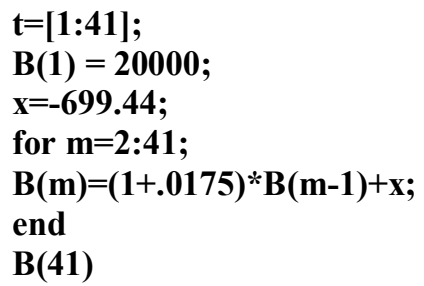

The output would beRs.0.0143, which is near to vanishing.

\section{Concluding Remarks}

The goal of the present article is to provide a tutorial exposition of the linear first order difference equation. Although it is less known to modelers in the behavioral and management sciences, DE(Difference Equation) is of fundamental importance in the theory of recursive relation or in discrete change. In this paper it is tried to provide a simple, intuitive explanation of the method so the reader can have a grasp of some of the basic principles. Ultimately, it is expected the reader will apply the method in his or her mathematical modeling efforts so a plethoraof widely available, DE-based analyses can be performed on data thereby extracting as muchinformation and insight as possible into the underlying mental process under investigation.

\section{References:}

[1] C. Ahlbrandt and A. Peterson, Discrete Hamiltonian Systems, Kluwer, Dordrecht, 1996. MR 98m:39043

[2] C. Brezinski, History of Continued Fractions and Pad e Approximants, Springer-Verlag,New York, 1991. MR 92c:01002

[3] B. Carlson, Algorithms involving arithmetic and geometric means, AMS Monthly 78(1971), 496-505. MR 44:479

[4] G. Dahlquist, Convergence and stability in the numerical integration of ordinary differential equations, Math. Scandinavica 4(1956), 33-50. MR 18:338d

[5] G. Dahlquist, 33 years of numerical instability, part 1, BIT 25(1985), 188-204. MR 86h:65006

[6] W. Gautschi, Zur Numerik rekurrenter Relationen, Computing 9(1972), 107-126. MR 47:1270

[7] J. Gleick, Chaos: Making a New Science, Penguin Books, New York, 1987. MR 91d:58152

[8] H. Goldstine, A History of Numerical Analysis from the 16th through the 19th Century,Springer-Verlag, New York, 1977. MR 58:4774

[9] P. Hartman, Difference equations: disconjugacy, principal solutions, Green's functions, complete montonicity, Trans. AMS 246(1978), 1-30. MR 80a:39004

[10] J. Wimp, Computation with Recurrence Relations, Pitman, Boston, 1984. MR 85f:65001

[11] Class notes AkilaWeerapana Fall Semester '07-08'

[12] Dr Ian Rudy (http://people.pwf.cam.ac.uk/iar1/contact.html) 